\title{
Conversion from Film Based Transfer Method Neutron Radiography to Computed Radiography for Post Irradiation Examination of Nuclear Fuels
}

\author{
Glen C Papaioannou ${ }^{1, a^{*}}$, Dr. Aaron E Craft ${ }^{2, b}$, Michael A Ruddell ${ }^{3, c}$ \\ ${ }^{1}$ Idaho National Laboratory, P.O. Box 1625, Idaho Falls, ID 83415, USA \\ ${ }^{2}$ Idaho National Laboratory, P.O. Box 1625, Idaho Falls, ID 83415, USA \\ ${ }^{3}$ Idaho National Laboratory, P.O. Box 1625, Idaho Falls, ID 83415, USA \\ aglen.papaioannou@inl.gov, baaron.craft@inl.gov, 'cmichael.ruddell@inl.gov
}

\begin{abstract}
Keywords: Transfer Method Neutron Radiography, Computed Radiography, Conversion Foils, Dysprosium, Indium, Image Plates, Image Resolution, Post Irradiation Examination, Nuclear Fuels, NRAD, Idaho National Laboratory
\end{abstract}

\begin{abstract}
The Idaho National Laboratory supports multiple programs that are actively developing, testing, and evaluating new nuclear fuels including advanced commercial nuclear fuels, accident tolerant fuels, reduced-enrichment research reactor fuels, transmutation fuels, and advanced reactor fuels. Post irradiation examinations (PIE) of nondestructive and destructive techniques are performed at INL to research nuclear fuel behavior and performance under various conditions. The Neutron Radiography Reactor (NRAD) at INL is utilized to perform nondestructive examinations of irradiated nuclear fuels and the results of these examinations are used to aid in the decision making process for subsequent destructive exams. NRAD has historically performed transfer method neutron radiography with film as the imaging medium. This paper documents the effort to convert from film to computed radiography for PIE. Equipment selection, system characterization, and testing were performed as part of phase I. Phase II, as described in this paper, focuses on determining image resolution of the computed radiography system.
\end{abstract}

\section{Introduction}

The current neutron radiography method used for evaluating irradiated fuel at NRAD is the foilfilm transfer technique [1]. Conversion foils (dysprosium and indium) absorb neutrons from a neutron beam that pass through the fuel and become temporarily radioactive in inverse proportion to their absorption in the fuel. The foils are then removed from the beam and film is placed in contact with the activated foil and exposed to the decay radiation from the foil overnight. After this exposure, the film is chemically-processed and scanned to produce the final radiographic image. This transfer technique is time consuming, but is one of very few techniques that can provide high quality radiographic images of irradiated fuel. INL is pursuing multiple efforts to advance its neutron imaging capabilities for evaluating irradiated fuel and other applications, including converting from film to photo-stimulated phosphor (PSP) image plates (IP) for neutron computed radiography (nCR). Neutron nCR is the current state-of-the-art for neutron imaging of highly-radioactive objects [2].

The $n$ CR process uses the transfer method but an IP is coupled to the activated foil instead of film (Figure 1). 


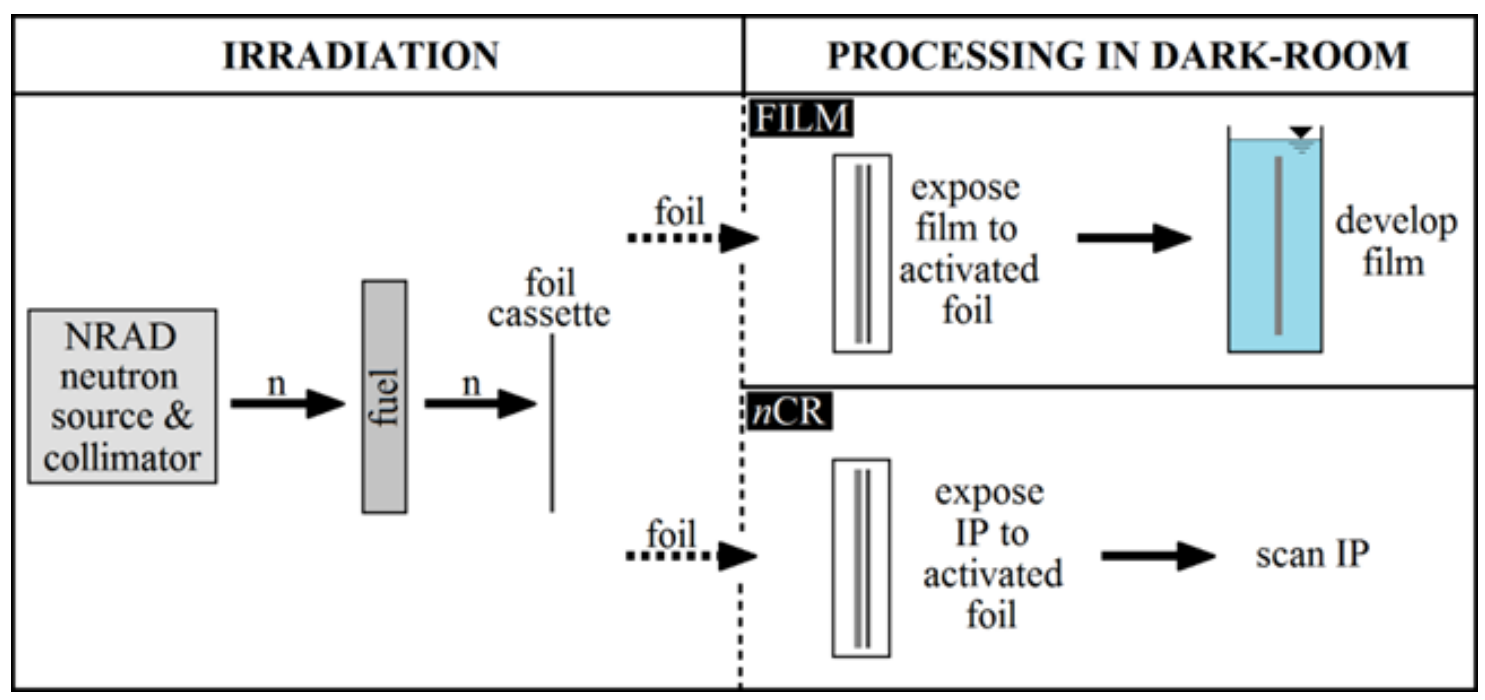

Fig. 1. Transfer Method Neutron Radiography Process

\section{Computed Radiography Equipment and Characterization}

A complete nCR system includes PSPs, IPs, an nCR scanner, a computer control workstation, and a high resolution computer monitor. nCR is based on PSP powder deposited on a substrate to form an IP. The substrate provides structural support for the IP. Information on the composition and structure of the phosphor layer is closely guarded by manufacturers and often proprietary, and thus largely unavailable. The protective layer protects the PSP from damage, and is an abrasion-resistant material that is transparent to the light-spectra of interest. The IPs chosen for this application are the flexible Carestream (formerly Kodak) plates, which were chosen based on their image quality and relatively robust protective layer. Commercial manufacturers typically provide three grades of IP: 1) standard or general purpose (GP), 2) high-resolution (HR), and 3) ultra-sharp or "blue" plate. The GP plates have a thicker PSP layer than the HR and blue plates, and thus they require less exposure dose at the expense of lower spatial resolution. HR and blue plates have a thinner PSP layer than GP plates, and thus require more exposure dose but provide higher resolution. The PSP layer in blue IPs contain a blue dye, which absorbs the red scanner laser, reducing the size of the laser interaction volume from which the blue light is emitted, producing higher sharpness in the resulting image. Different commercial manufacturers of IPs may use different substrate materials, have differing PSP chemistries and thicknesses, and use different concentrations of blue dye in the blue IPs, all generally provide the same three grades of IPs. The GP plates provide adequate spatial resolution for general purpose applications, in the range of $350-400$ microns. If higher spatial resolution is required than the GP plates can provide, the HR plates may be desirable, 250 microns. While the HR plates offer improved spatial resolution, they require almost twice the exposure dose compared to GP plates. Blue plates offer higher resolution capabilities than even the HR IPs, 175 microns, but require around 60\% more exposure dose compared to the HR plates to produce the same output signal. When comparing these plates to decide which type of IP is most appropriate to use, the desired image quality and exposure times, including both the neutron beam exposure and decay times should be the determining factors.

There are a variety of CR scanners available from commercial manufacturers, and their specific features may vary widely. Two styles of scanners include flat-bed scanners where the IP is flat while scanned and drum-type scanners where the IP is curved, but the fundamental mechanism of acquiring the image signal from an exposed IP is the same regardless of scanner 
style. A mechanical transport mechanism moved an exposed IP through the scanner. A red laser rasters over the surface of the IP and stimulates the activated PSP, which then releases the stored energy as blue light through photo-stimulated luminescence. The blue light signal is amplified by a photo-multiplier tube into a measureable signal, then converted to a digital signal that is read by a computer. The position of the laser and the output signal are both known, allowing for the formation of an image. Many scanners provide an erasure function that erases the IP using an array of red LEDs positioned after the scanner laser. The laser spot size is often the driving factor for the effective spatial resolution that a scanner can provide. Scanners with $50 \mu \mathrm{m}$ diameter laser spot size are commonly available, and high-resolution scanners with laser diameters of around $10 \mu \mathrm{m}$ are also available. The scan resolution is determined by the mechanical transport speed and the laser rastering rate. The PMT voltage may be manipulated to amplify the signal from an IP to utilize the bit-depth provided by the scanner. Manipulation of these and other scanner parameters may be available to the operator depending on the particular software and manufacturer.

Conversion to $n \mathrm{CR}$ consists of characterization and qualification of an $n \mathrm{CR}$ system using the current foil transfer technique with the goal of maintaining similar image quality compared to the current technique with film. Preliminary studies measured the image quality of radiographs acquired using the existing film-based method and determined CR system equipment capable of providing the desired image quality. The hardware selected for this application included a ScanX Discover HR scanner made by ALLPRO Imaging and image plates from Carestream.

Neutron radiography takes significantly more resources (time and cost) than x-ray radiography, and acquisition of multiple radiographs is required to test and characterize the response of the CR system to various input parameters. As part of phase I, x-ray characterization of the CR system was performed to provide a better understanding of the CR system and inform subsequent image acquisitions using neutrons. Additionally, $\mathrm{x}$-ray CR $(x \mathrm{CR})$ was performed to provide a baseline for the performance of the scanner that could be used in the future to test whether the scanner is still performing to its capacity or if maintenance is required.

\section{Neutron Computed Radiography of Irradiated Fuel}

A set of nCR radiographs were taken of an irradiated fuel pin, and the IPs were scanned and the grayscale intensities of the resulting images evaluated. From these intensities, the change in PMT voltage required to achieve the desired intensity was calculated. The second set of exposed IPs was scanned using these calculated values provided the expected grayscale values. Thus, $\mathrm{x}$-ray characterization provided the understanding needed to significantly reduce the number of neutron radiographs required to determine the scanner settings for nCR.

Figure 2 and 3 shows neutron radiographs of an irradiated fuel pin acquired using film and nCR with a XL-Blue plate and an HR plate. The exposure parameters for these radiographs are listed in Table 1 and were performed at an L/D of 125 . The histograms of these images have been adjusted to display a similar grayscale range to allow for a more meaningful comparison between film and nCR. From a qualitative perspective, the image quality of nCR closely matches that of film, and features such as cracks and gaps can be visualized with similar contrast. Film radiographs exhibit higher sharpness than nCR but lower latitude for the thermal-neutron radiographs. 
Table 1. Image acquisition parameters: L/D 125, 5 half-life decay (overnight)

\begin{tabular}{|c|lccc|}
\hline Conversion Foil & Imaging Medium & $\begin{array}{c}\text { Neutron Beam } \\
\text { Exposure Time } \\
\text { (min.) }\end{array}$ & $\begin{array}{c}\text { PMT } \\
\text { Voltage }\end{array}$ & $\begin{array}{c}\text { Scan } \\
\text { Resolution }\end{array}$ \\
\hline $\begin{array}{c}\text { Dysprosium } \\
125 \mu \mathrm{m} \text { thick metal } \\
\text { foil } \\
\text { (unfiltered neutron } \\
\text { beam) }\end{array}$ & AGFA D3-SC film & $22: 00$ & - & $47 \mathrm{lp} / \mathrm{mm}$ \\
\hline $\begin{array}{c}\text { Indium } \\
125 \mu \mathrm{m} \text { thick metal } \\
\text { foil }\end{array}$ & Kodak T200 & $20: 00$ & $625 \mathrm{~V}$ & $25 \mu \mathrm{m}$ \\
$\begin{array}{c}\text { (cadmium-filtered } \\
\text { beam) }\end{array}$ & $n$ CR, HR plate & $22: 00$ & - & $25 \mathrm{lp} / \mathrm{mm}$ \\
\hline
\end{tabular}
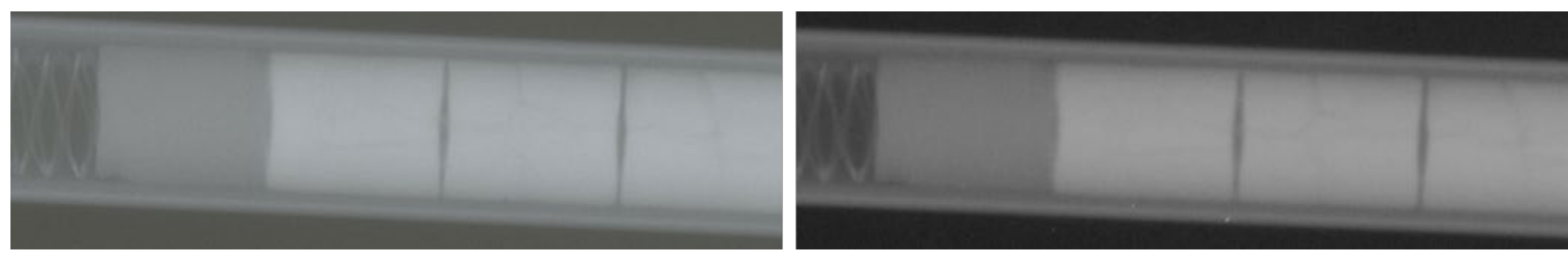

Fig. 2. Thermal neutron radiographs of an irradiated fuel pin acquired using film (left) and $n C R$ with XL-Blue plate (right)

Fig. 3. Epithermal neutron radiographs of an irradiated fuel pin acquired using film (left) and nCR HR plate (right)

\section{Determining Image Resolution}

To determine the resolution of the NRAD system utilizing CR, a new Resolution Test Piece, RTP, was designed for use in the NRAD elevator. The new design consists of several standards to provide qualitative and quantitative image resolution. A Siemens Star furnished by the Paul Scherrer Institute for resolution determination in multiple directions, with the outer ring starting at 500 microns and the inner ring measuring 20 microns. See Figure 4. Edge specimens positioned at 2-5 degrees, one of gadolinium and one of hafnium for MTF determination from thermal and epithermal neutron energies, respectfully. Gadolinium doped, dimensionally inspected rulers mounted in the $\mathrm{X}$ and $\mathrm{Y}$ directions. Lastly, a beam purity indicator and a sensitivity indicator per ASTM E545 neutron radiography image quality indicators. 


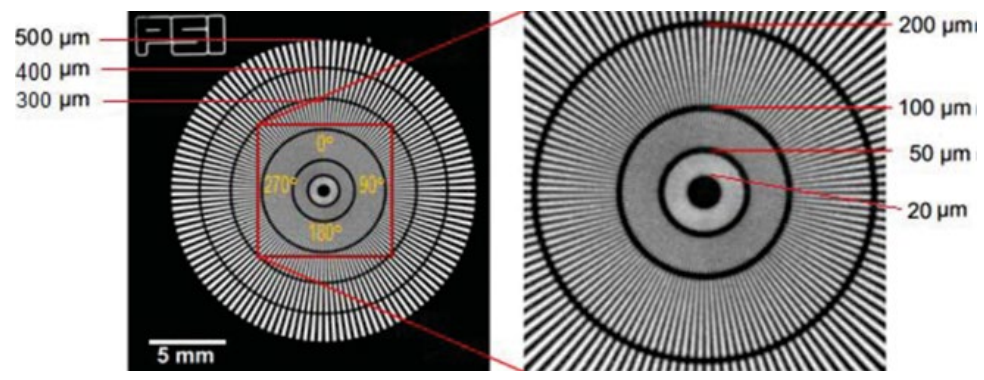

Figure 4 Siemens Star

The RTP was positioned remotely in the beamline to acquire baseline images, measure MTF, visible resolution, and contrast. Several exposures were taken with film and nCR for evaluation, see Figure 5. Images of the sensitivity indicator exhibit a Category-I facility according to ASTM E-545 [3, 4]

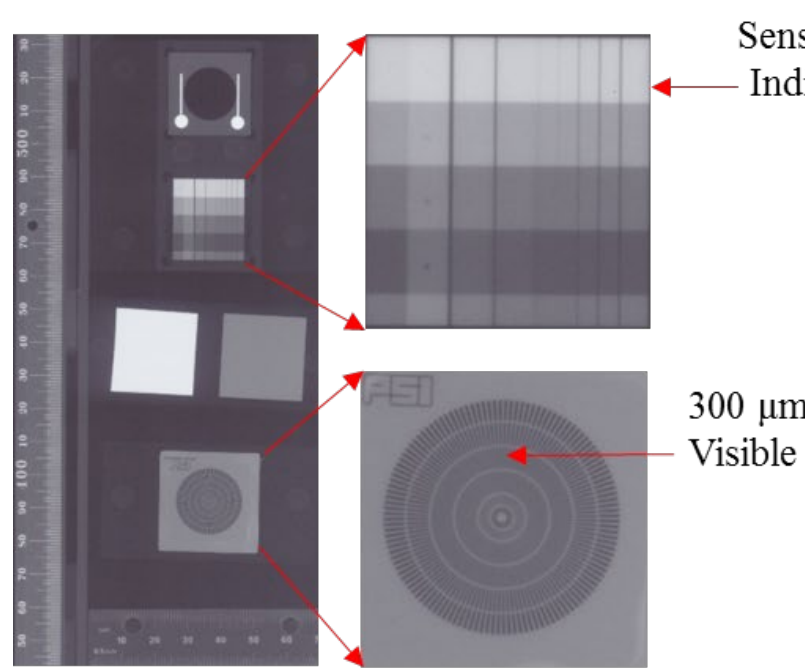

D3-SC Film RTP Image, Thermal

Neutron Energy, from

Dysprosium Transfer Foil

Figure 5 Resolution Test Piece

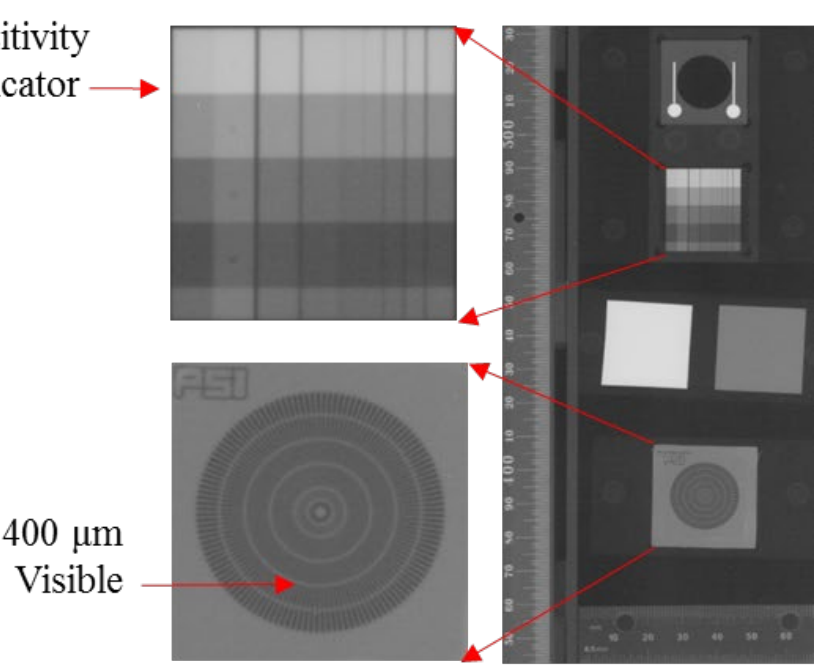

nCR Blue Plate RTP Image,

Thermal Neutron Energy, from

Dysprosium Transfer Foil

It is important to note that the transfer method of irradiated nuclear fuels requires shielding and confinement. These factors determine the maximum resolution possible for the given system due to geometric unsharpness. That is the distance from the specimen to the conversion foil. Irradiated specimens are contained inside a hot cell, and lowered down and elevator shaft into the neutron beam. The shaft acts as confinement for the irradiated specimen, and is located inside a shielded subcell room, similar to an X-ray cave. The transfer foil is remotely positioned outside the elevator shaft, as close as possible to the shaft wall. Based on the irradiated specimen size, shape, and fixturing, the distance between the transfer foil and the specimen can range upwards of $10 \mathrm{~cm}$. The further away the specimen is from the foil, the more out of focus it will appear in the image negatively impacting the image resolution.

\section{Summary}

The final qualification of the nCR system requires written justification containing system characterization results and baseline image interpretation between film and nCR of image quality indicators and irradiated fuel experiments. Data from phase I characterization efforts and phase II image analysis will be documented in a report stating the range of operating parameters and resolution of the system. Currently, the NRAD nCR system is capable of 400 micron visible 
resolution. Continuing efforts directed at improving resolution involve; improving geometric unsharpness with new specimen handling fixtures that can be positioned closer to the transfer foil, increasing L/D ratio to 300, and improving foil surface geometry to enhance contact with the IP during decay.

\section{References}

[1] Craft, A.E., Chichester, D.L., Williams, W.J., Papaioannou, G.C., and Wachs, D.M., 2015. Conversion from radiographic film to photo-stimulable phosphor plates for neutron computed radiography of irradiated nuclear fuel. ASNT Annual Conference 2015, pp. 23-27.

[2] Vontobel, P., Tamaki, M., Mori, N., Ashida, T., Zanini, L., Lehmann, E. H., \& Jaggi, M., 2006. Post-irradiation analysis of SINQ target rods by thermal neutron radiography. Nuclear Materials, 356(1), 162-167. https://doi.org/10.1016/j.jnucmat.2006.05.033

[3] Craft, A.E., Hilton, B.A., Papaioannou, G.C., 2015. Characterization of a neutron beam following reconfiguration of the Neutron Radiography Reactor (NRAD) core and addition of new fuel elements. Nuclear Engineering and Technology, Submitted for publication. https://doi.org/10.1016/j.net.2015.10.006

[4] ASTM International, 2005. Standard method for determining image quality in direct thermal neutron radiographic examination. American Society for Testing and Materials, ASTM E545-05. 